\title{
Making Use Of What You Don't See: Negative Information In Markov Localization*
}

\author{
Jan Hoffmann, Michael Spranger, Daniel Göhring, and Matthias Jüngel \\ Department of Computer Science \\ Artificial Intelligence Laboratory \\ Humboldt-Universität zu Berlin \\ Unter den Linden 6 \\ 10099 Berlin, Germany \\ http://www.aiboteamhumboldt.com
}

\begin{abstract}
This paper explores how the absence of an expected sensor reading can be used to improve Markov localization. This negative information usually is not being used in localization, because it yields less information than positive information (i.e. sensing a landmark), and a sensor often fails to detect a landmark, even if it falls within its sensing range. We address these difficulties by carefully modeling the sensor to avoid false negatives. This can also be thought of as adding an additional sensor that detects the absence of an expected landmark. We show how such modeling is done and how it is integrated into Markov localization. In real world experiments, we demonstrate that a robot is able to localize in positions where otherwise it could not and quantify our findings using the entropy of the particle distribution. Exploiting negative information leads to a greatly improved localization performance and reactivity.

Index Terms-Negative Information, Negative Evidence, Mobile Robots, Markov Localization, Monte Carlo Localization, Entropy
\end{abstract}

\section{INTRODUCTION}

The classic example of negative information was described in the Sherlock Holmes case "Silver Blaze." In this case, a house has been broken into. Under such circumstances, one would expect the watch-dog to bark. The curious incident of the non-barking of the dog in the nighttime provides Holmes with the information that the dog must know the burglar, allowing him to solve the case. Applied to mobile robot localization, this means that conclusions can be drawn from expected but actually missing sensor measurements [5]. Markov localization methods, in particular Monte Carlo localization, have proven their power in numerous robot navigation tasks, e.g. in office environments [1], in the museum tour guide Minerva [14], in the highly dynamic RoboCup environment [8], and outdoor applications in less structured environments [10]; an evaluation of the various algorithmic approaches is given in [3].

Our work is focussed on localization based on landmarks. Whenever a robot senses a landmark, the localization estimate is updated using the sensor model. This sensor model is acquired before the actual run. It describes the probability of the measurement $z$ given a state $s$ (position, orientation, etc.)

${ }^{*}$ The project is funded in part by the German Research Foundation (DFG), SPP 1125 "Cooperative Teams of Mobile Robots in Dynamic Environments". of the robot. Sensor updates only occur when landmarks are detected. If no landmark is detected, the state estimation is updated using (only) the motion model of the robot.

Example. Consider a robot driving down a corridor as shown in fig. 1a-1d. The robot has a sensor to detect doors when it is standing in front of one. Let us assume further that the robot is moving to the right but is oblivious of its starting position. As it starts to move to the right it passes and senses a door. Given this information, it could be standing in front of either of the doors (states $s_{\text {left }}$ and $s_{\text {right }}$ ). As it moves on, it does not pass another door for some time. At time $t=t_{3}$, if $s_{\text {left }}$ had been the true position, the robot would have had passed another door by now. Using the negative information of not perceiving a door, the belief based on $s_{\text {left }}$ can be ruled out. As Thrun, Bugard, and Fox put it quite graphically, "not seeing the Eiffel Tower in Paris implies that it is unlikely that we are right next to it" [13].

We present a localization approach that incorporates such negative information. To our knowledge, no explicit study of using negative information in Markov localization has been published. One difficulty is brought about by the fact that, generally speaking, sensing a landmark constitutes a greater information gain than not sensing one simply because there are many positions within the robot's environment from where the landmark cannot be perceived. A landmark is, by definition, something that stands out in an environment.

The other difficulty in implementing a system that uses negative information on a real robot is that there are two main reasons for the absence of an expected sensor reading: the target may not be there or the sensor may simply be unable to detect the target (due to occlusions, sensor imperfections, imperfect image processing, etc.). Differentiating the two cases is not a trivial task and requires careful sensor modeling. We address this problem by considering the field of view of the robot and by using obstacle detection to estimate occlusions.

Negative information modeling has been applied to object tracking (see [12] for an introduction and [5] for an overview). The event of not detecting an object is treated as evidence that can be used to update its probability density function [6]. In the RoboCup domain, not seeing the ball on the field 
can be used to delete Monte Carlo particles in that region as long as occlusions are considered [7]. Negative information is also mentioned in the context of simultaneous localization and mapping (SLAM) where it is used to adjust the confidence in landmark candidates [10].

Outline. In section II we will show how negative information can be incorporated into Monte Carlo localization. We will then extend the sensor model by also modeling the probability of non-detection events. In section III the positive impact on localization will be shown in simulation and real world experiments using the Sony Aibo ERS-7 robot.

\section{ExPloiting NegATIVE INFORMATION}

\section{A. Iterative Bayesian Updating}

This work is based on Markov localization for mobile robots as described in [1], [13], [11]. The belief state of the robot $\operatorname{Bel}\left(s_{t}\right)$ at time $t$ to be in state $s_{t}$ is determined by all previous robot actions $u_{t}$ and observations $z_{t}$. Using Bayes law and the Markov assumption, $\operatorname{Bel}\left(s_{t}\right)$ can be written as a function depending only on the previous belief $\operatorname{Bel}\left(s_{t-1}\right)$, the last robot action $u_{t-1}$, and the current observation $z_{t}$ :

$$
\begin{aligned}
\operatorname{Bel}^{-}\left(s_{t}\right) & \longleftarrow \int p\left(s_{t} \mid s_{t-1}, u_{t-1}\right) \operatorname{Bel}\left(s_{t-1}\right) d s_{t-1} \\
\operatorname{Bel}\left(s_{t}\right) & \longleftarrow \eta p\left(z_{t} \mid s_{t}\right) \operatorname{Bel}^{-}\left(s_{t}\right)
\end{aligned}
$$

with normalizing constant $\eta$. Equation 1 shows the a priori belief $\mathrm{Bel}^{-}\left(s_{t}\right)$ which propagates the previous belief using the motion model of the robot. The measurement is then incorporated into the belief as described in (2) using the sensor model ('sensor updating').

In Markov localization, given an initial belief $\operatorname{Bel}\left(s_{0}\right)$ at $t=t_{0}$, the robot first updates its belief using odometry and then incorporates new sensor information. The belief is updated iteratively in this fashion for every following time step. In the absence of sensor readings, no sensor updating is performed and the belief is updated solely using odometry.

\section{B. The Notion Of Negative Information}

Negative information describes the absence of a sensor reading in a situation where a sensor reading is expected given the current position estimate.

To integrate negative information, imagine a binary sensor being added that fires whenever the primary sensor does not detect a particular landmark $l$. Its probability of it firing is given by:

$$
p\left(z_{l, t}^{\star} \mid s_{t}\right)
$$

This sensor model can be used to update the robot's belief whenever it fails to detect a landmark, i.e. when negative evidence is acquired. Fig. 2 shows the probability $p\left(z_{t}^{\star} \mid x_{t}, y_{t}\right)$ of not sensing a landmark on a RoboCup field at position $\left(x_{t}, y_{t}\right)$ summed over all possible robot orientations. This figure also shows that it is most likely for the robot to sense a landmark when it is standing in the middle of the field. The
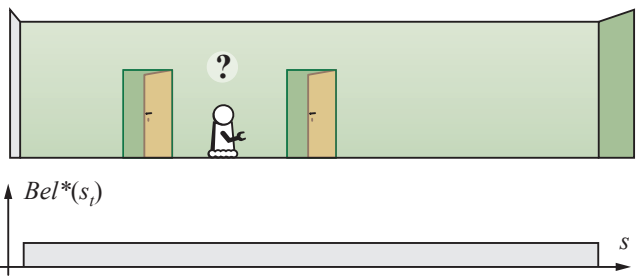

Fig. 1a. $\left(t=t_{0}\right)$ Illustration of a robot localizing in an office hallway. The robot has a sensor to detect doors. At the beginning, the robot does not know its position in the hallway (uniform belief distribution $\mathrm{Bel}^{\star}\left(s_{t}\right)$ ). At this time, no sensing of the world takes place.

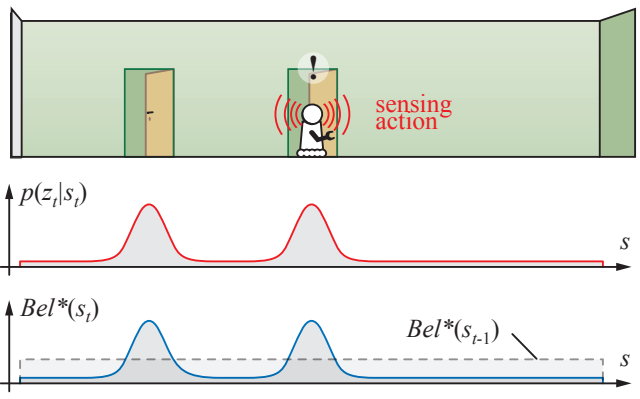

Fig. 1b. $\quad\left(t=t_{1}\right)$ The robot has moved down the hallway and now senses a door $p\left(z_{t} \mid s_{t}\right)$ which results in the shown belief $B e l^{\star}\left(s_{t}\right)$. It has two peaks since the robot could be standing in front of either door. The previous distribution is illustrated by the dashed line.
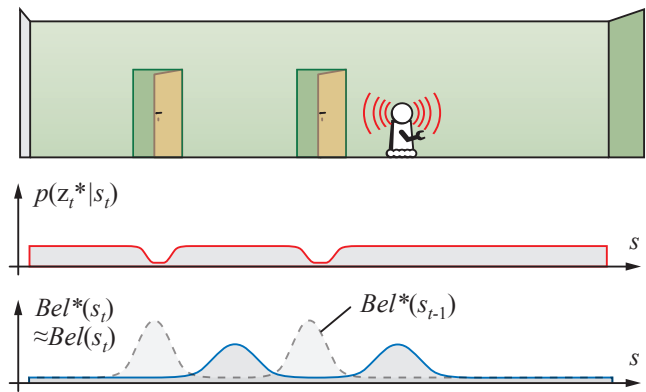

Fig. 1c. $\left(t=t_{3}\right)$ The robot moves on. There are no doors nearby so the "door sensor" does not sense a door. The sensor update distribution is shown in $p\left(z_{t}^{\star} \mid s_{t}\right)$. This negative information is of negligible use at this position: it does not help differentiate between the peaks.
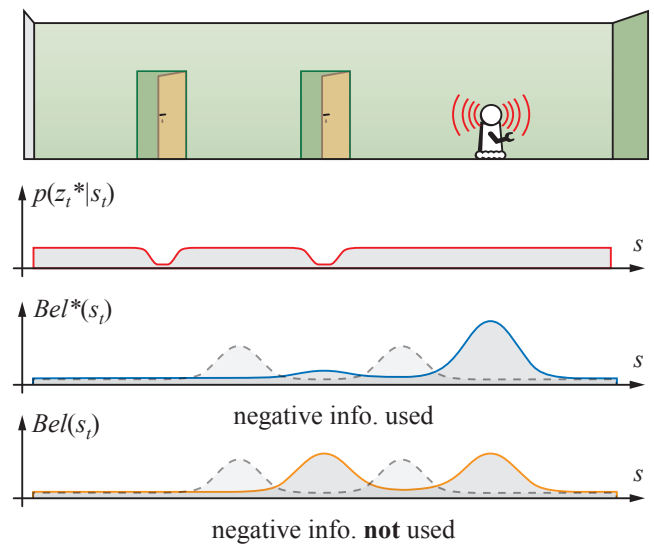

Fig. 1d. $\left(t=t_{4}\right)$ The robot moves on and the door sensor still does not sense a door. $\mathrm{Bel}^{\star}\left(s_{t}\right)$ shows the belief if negative information is taken into account, whereas $\operatorname{Bel}\left(s_{t}\right)$ shows the belief without using negative information to better illustrate the case. As can be seen from the diagram, using negative information allows the robot to rule out the left peak. 


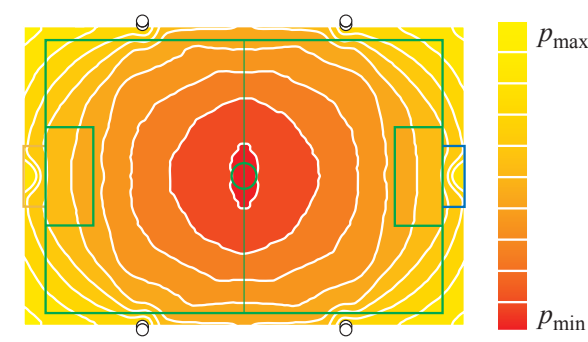

Fig. 2. Probability of not sensing a landmark for a robot on a RoboCup soccer field. For a robot located around the center of the field, it is hard to miss landmarks.

likelihood of not sensing a landmark is highest for positions at the edge of the field as the robot may be facing outwards.

This rather coarse way of incorporating negative information can be refined by taking into account the sensing range $r_{t}$ of the robot's sensors and possible occlusions $o_{t}$ of landmarks. The sensing range is the physical volume that the sensor is monitoring. In case of a stationary robot, $r_{t}=r_{0}$ is constant, for a mobile robot with a pan-tilt camera it is not. By $o_{t}$ we denote a means of detecting whether or not occlusions have occurred. In practice, this can be calculated from a map of the environment, directly sensed by a sensor such as a laser range finder, or derived from a model of moving objects in the environment.

Combining the two yields the probability of not sensing an expected landmark $l$ at time $t$ :

$$
p\left(z_{t, l}^{\star} \mid s_{t}, r_{t}, o_{t}\right)
$$

Whenever a landmark is not detected, it can be used in the sensor update step of the Iterative Bayesian Updating (see Algorithm 1).

\section{Sensor Modeling For The Sony Aibo}

1) Field of View: The ERS-7 is a legged robot with a camera mounted in its head. The camera has a horizontal opening angle of $55^{\circ}$ and the robot's head has 3 degrees of freedom (neck tilt, head pan, head tilt). We abbreviate gaze direction by $\varphi=\left(\varphi_{\text {tilt } 1}, \varphi_{\text {pan }}, \varphi_{\text {tilt } 2}\right)$. The sensing range is calculated by considering the field of view (FOV) of the robot:
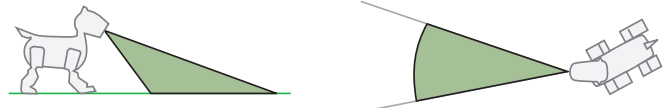

2) Occlusion: In order to account for occlusions, we opted for an approach that has been used successfully for detecting obstacles, referred to as 'visual sonar' [4], [9]: The camera image is scanned in vertical scan lines and unoccupied space in the plane of the field is detected since it can only be of green or white color (field lines). Scanning for these colors tells the robot where obstacles are and where there is free space which in turn can be used to determine if the visibility of the landmark is impaired, i.e. if it is occluded by other robot or some other obstacle. More specifically, if the expected

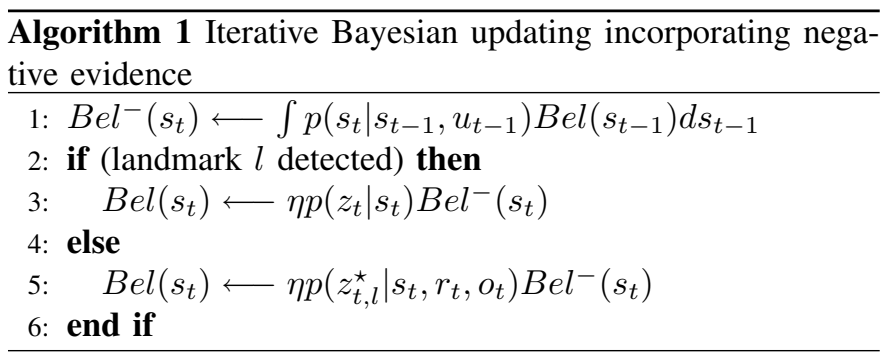

landmark lies in an area where the robot has detected free space, the likelihood of the corresponding pose estimate is decreased. If it lies outside of the detected free space, no inference can be made.

Taking FOV and occlusion into account, the sensor model for not perceiving an expected landmark (equation 4) becomes:

$$
p\left(z_{t, l}^{\star} \mid s_{t}, z_{t, \mathrm{obs}}\right)
$$

Where $z_{t, \text { obs }}$ describes the current obstacle percept and $s_{t}=\left(x_{t}, y_{t}, \vartheta_{t}, \varphi_{t}\right)$ the robot state consisting of the robot pose (position $x_{t}, y_{t}$, and orientation $\vartheta_{t}$ ) and the current gaze direction $\varphi_{t}$.

\section{EXPERIMENTAL RESULTS}

The RoboCup Sony 4-Legged League serves as a test bed for our work. In the 4-Legged League, teams of 4 Sony Aibo ERS-7 robots play soccer against each other in a color coded environment (see the official RoboCup web site for details: www.robocup.org). Colored beacons (4 uniquely color coded beacons plus a blue and a yellow goal) and the field lines (similar to the real soccer field lines) serve the robots for localization. In our experiment, unless otherwise stated, only landmarks were used for localization to emphasize the effect of using negative information.

\section{A. Monte Carlo Localization, Implementation}

This work is based on the Monte Carlo localization described in [11] which also serves as a base line implementation. Sensor updating was extended to account for FOV and occlusion as described. This also requires sensor updating to be triggered by new camera images regardless of whether or not there was a percept. Before re-sampling, the weight of an individual particle is calculated as follows: Of all landmarks $L$, the subset of landmarks $L^{\prime}$ is detected, the subset $L^{\star}$ is expected but not detected, and lastly the subset $L^{\diamond}$ is not detected but was also not expected: $L=L^{\prime} \cup L^{\star} \cup L^{\diamond}$ and $L^{\star} \cap L^{\prime}=\emptyset$. The probability of a particle $p_{i}$ is calculated by multiplying all the likelihoods of all gathered evidences:

$$
p_{i}=\underbrace{\prod_{l \in L^{\prime}} s_{l}\left(\alpha_{\text {measd }}, \alpha_{\text {expd }}\right)}_{\text {detected }} \cdot \underbrace{\prod_{l \in L^{\star}} s_{l}^{\star}\left(\varphi, \alpha_{\text {expd }}\right)}_{\text {expected and not detected }}
$$

The function $s_{l}$ is an approximation of the sensor model and returns the likelihood of sensing the landmark $l$ at angle $\alpha_{\text {measd }}$ for a particle $p_{i}$ that expects this landmark to be at 


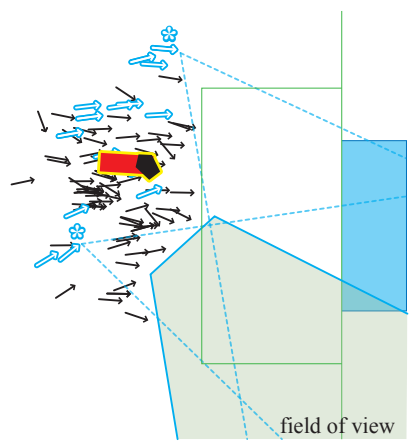

1)

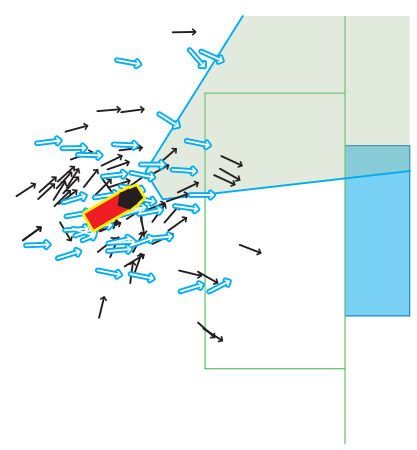

2)
Fig. 3. Incorporating negative information. White (outlined) arrows denote particles that receive negative information and are therefore less likely than others, i.e. their weights are being updated by negative evidence. In (1), the effect of using negative information is shown for a robot that is well localized and frequently sees landmarks. (2) Distribution shortly after the robot has been displaced (kidnapped): particles facing the goal are less likely and will eventually be eliminated from the distribution.

$\alpha_{\text {expd }}$. Function $s_{l}^{\star}$ models the probability of not sensing the expected landmark $l \in L^{\star}$ given the current sensing range as determined by $\varphi$, the robot pose associated with $p_{i}$, and the bstacle percept $z_{\text {obs }}$.

\section{B. Preliminary Experiment}

For illustration purposes, we conducted a preliminary experiment in simulation. In this experiment, the robot starts out being well localized and is then displaced to a position where it is not able to get any new sensor information (fig. 3). It is similar to the kidnapped robot problem, but here we emphasize the moment right after the robot is displaced rather than investigating how fast it can recover. The effect of the displacement on the Monte Carlo particle distribution is the following: particles which represent the previous belief become less likely when negative information is taken into account (i.e. the information that the landmark is not detected where it is expected). The distribution diverges towards particles which were less likely prior to the displacement. Particles representing the previous belief are eventually eliminated from the distribution because they are inconsistent with the current (negative) sensor data. Particles which differ from the previous belief just enough to be compatible with the current sensor data are favored; particles remain close to where the robot was last able to localize. This does, in most cases, better represent what has happened to the robot than distributing the particles uniformly over the entire field.

\section{Localization Experiment}

The following experiment is a localization task using the real robot. The robot is placed on the field at the location indicated in fig. 4, facing outwards. The robot performs a scanning motion with its head (pan range $\left[-45^{\circ}, 45^{\circ}\right]$ ) but does not move otherwise. From its position, it can only see one landmark. A panorama composed of actual robot camera images is shown in fig. 5. The a priori belief is assumed uniform. This position was chosen because it is a

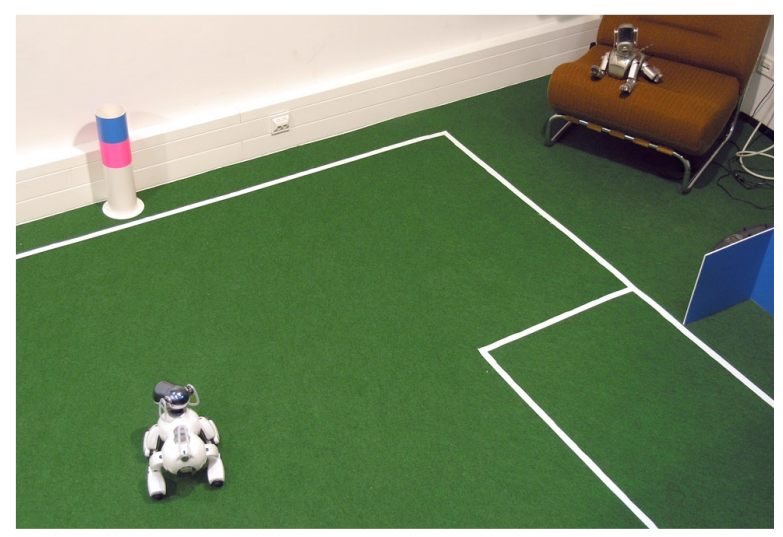

Fig. 4. Experimental setup: Robot is standing at the position shown in the photo. It performs a scanning motion with its camera.

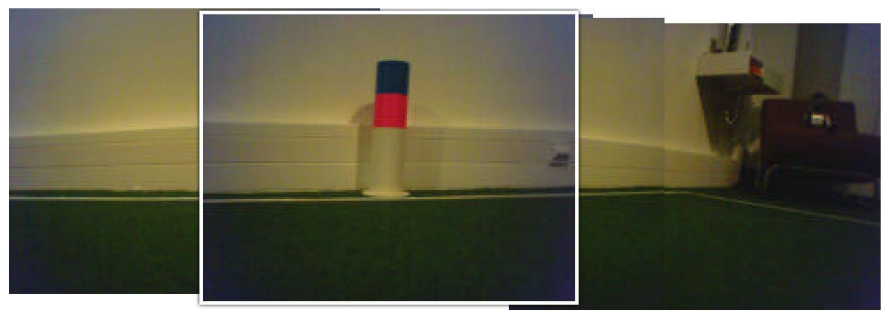

Fig. 5. A panorama view generated from actual camera images, single camera image highlighted. The robot can only see one landmark.

particulary difficult spot for the robot to localize given the limited sensor information. Two quantities can be used when a landmark is seen: its size in the camera image can be used to estimate the distance to the landmark $d_{l}$ and the relative angle to the landmark (bearing, $\alpha_{l}$ ) can be calculated from its position within the image. In practice we only use the bearing because the distance measurement is error prone. Using just the bearing, only the orientation of the robot can be inferred. Note that this differs from triangulation where distances are used.

In the following paragraphs, the basic localization not using negative information and localization incorporating negative information are compared. We first qualitatively analyze the particle distribution and then show how the entropy of the distribution decreases when negative information is considered.

1) Particle Distribution: The basic experiment was conducted using 100 particles for Monte Carlo localization. It was repeated on a log file containing camera images, robot joint angles, and odometry data using an increased particle count of 2000 to get a better representation of the probability distribution.

Not using negative information. Without using negative information, the robot is unable to localize (fig. 6). Only the orientation of the particles is adjusted according to the sensor readings. The apparent clustering in the small sample set in fig. 6 is not stable and, even after considerable time, the particles do not converge. The distribution for the larger sample set is uniform (w.r.t. position).

Note that the distribution is not circular because the distance 


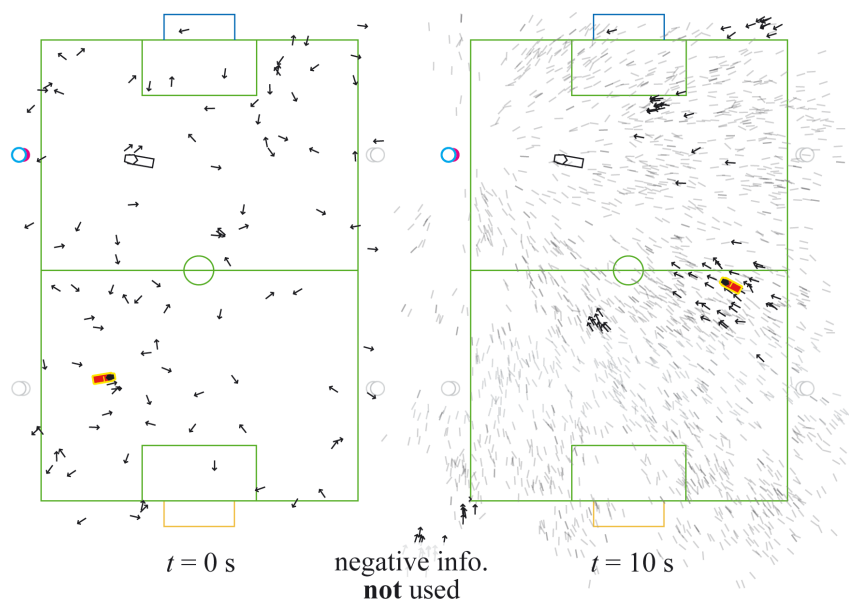

Fig. 6. Particle distribution not using negative information, initial uniform distribution and distribution after 10s. Solid arrows indicate Monte Carlo particles (100). The experiment was repeated using 2000 particles (shaded lines) to better represent the actual probability distribution. The actual robot position is indicated by the white symbol, the estimated robot pose by the solid symbol. Not using negative information and only using the bearing to the landmark, the robot is unable to localize. Some clusters of particles form but they do not converge. As one would expect, the position distribution is almost uniform but the relative angle is quite distinct.

to the landmark was not used. Instead, only the bearing to the landmark was used. This results in a radial distribution resembling magnetic field lines.

Incorporating negative information. The negative information gained in this experiment is not seeing but one landmark within the pan range (pardon the double negation). Incorporating this information, the robot is able to localize quickly. On average, the robot is reasonably well localized after about 10 secs with a pose error of less than $\Delta p=\left(25 \mathrm{~cm}, 25 \mathrm{~cm}, 20^{\circ}\right)$.

2) Entropy: We use the expected entropy $H$ as an information theoretical quality measure of the position estimate $\operatorname{Bel}\left(s_{t}\right)[2]:$

$$
H_{p}\left(s_{t}\right)=-\sum_{s_{t}} \operatorname{Bel}\left(s_{t}\right) \log \left(\operatorname{Bel}\left(s_{t}\right)\right)
$$

The sum runs over all possible states. The entropy of the particle distribution becomes zero if the robot is perfectly localized in one position, maximal values of $H$ mean that $\operatorname{Bel}\left(s_{t}\right)$ is uniformly distributed.

Fig. 8 shows the progression of the distribution's entropy over time for the above localization experiment calculated from the 100 particle distribution.

Not using negative information. The experiments starts with a uniform particle distribution which equals to maximum entropy. When the landmark comes into view, a decrease in entropy is observed. This information gain is due to the robot being able to now infer its relative orientation w.r.t. the landmark. Since there are no constraints on the robot's position, the entropy remains at a relatively high level. This is easily seen by separately calculating the entropy of the angle and position distributions. Note that even though there is a drop in entropy, the pose estimate itself is still highly uncertain.

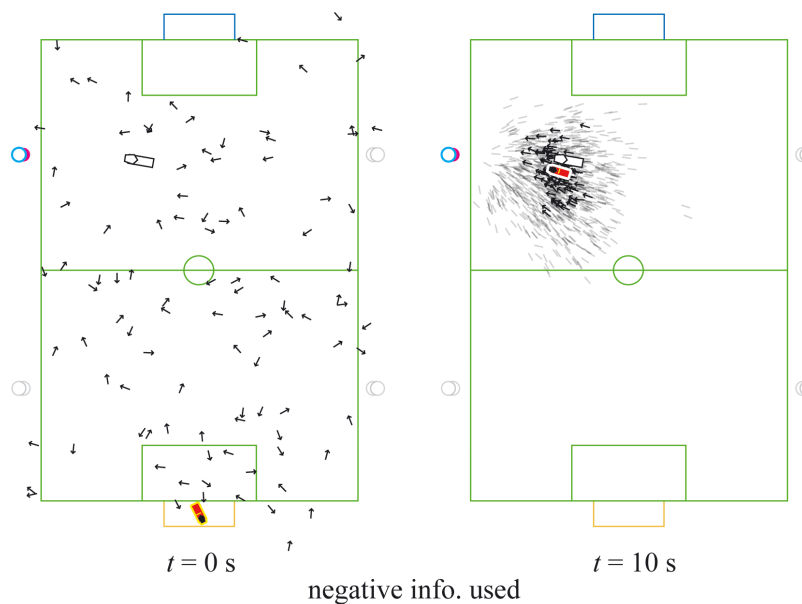

Fig. 7. Particle distribution when negative information is incorporated, initial uniform distribution and distribution after $10 \mathrm{~s}$. When incorporating negative information, the robot is able to localize quickly.

Incorporating negative information. When using negative information, the entropy decreases even before the first sensor reading. The information gain is much smaller than that caused by perceiving a landmark but nevertheless noticeable. As soon as there is a percept, the negative information in combination with the knowledge of the robot's orientation results in a quick convergence towards the actual robot pose. This is remarkable since without using negative information, localization was not possible.

Using field lines for localization. The previous experiment was repeated using field lines for localization in addition to landmarks. This enables the robot to localize quickly at the actual robot pose even when using the basic localization (fig. 8, right). Adding negative information, however, greatly increases the rate of convergence and the overall level of entropy is reduced even further. The decrease of entropy when incorporating negative information is not obscured by the usage of lines for localization although field lines offers a much greater information content than negative information.

Kidnapped Robot. The kidnapped robot problem is a commonly used benchmark for the flexibility and robustness of localization algorithms [3]: a localized robot is displaced and the time for it to recover is measured. Our kidnapped robot experiments underlined and confirmed the already stated findings. The robot is able to recover from displacements without using negative information as soon as it successively sees three landmarks. In regions where this was not guaranteed, the case is different. Whereas without using negative information, the robot does not have enough evidence to update its belief, incorporating negative information allows the robot to localize quickly and reliably in such regions.

The ability to localize more quickly using negative information is highly beneficial in real world applications where the robot is trying to actually perform a task rather than to localize perfectly. Such tasks often require the robot to focus its attention on objects other than landmarks and the 

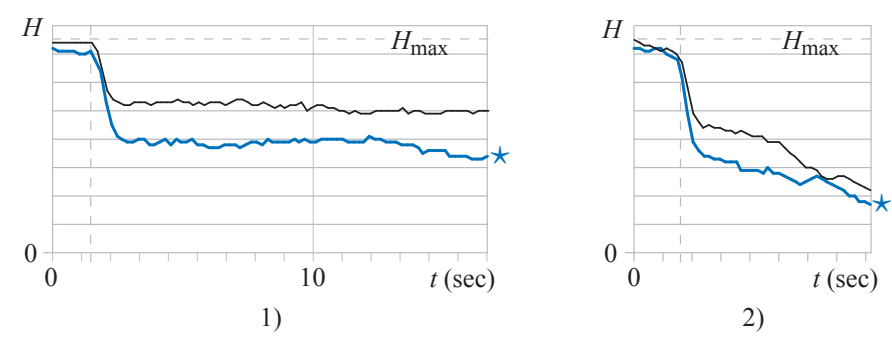

Fig. 8. Expected entropy of the belief in the localization task with $\left(^{\star}\right)$ and without (thin line) using negative information. 1) At first the robot does not see the landmark. As soon as the landmark comes into the robot's view (indicated by the dashed vertical line), the entropy drops. Using negative information, the quality of the localization is greatly improved and the entropy continues to decrease over time. 2) Additionally using field lines for localization enables the robot to localize even without negative information. Incorporating negative information, however, yields a higher rate of convergence and the entropy is significantly lowered.

sensing strategy may keep it from seeing as much of the world as it potentially could. Integrating negative evidence thus allows for more efficient sensing and improves overall robot performance.

\section{CONCLUSION}

We demonstrate the power of integrating negative information - the absence of an expected sensor reading - into Markov localization. Because sensors are more likely to overlook observable landmarks than hallucinate ones that are not visible, extra care has to be taken in designing the sensor model. To avoid false negatives, the model needs to take into account the sensor's sensing range and possible occlusions of landmarks. We present how such modeling can be achieved in general and specifically for a Sony Aibo robot in the RoboCup environment. In real robot experiments, we show that using negative information, a robot is able to localize in positions where it otherwise would not have been able to localize. The robot senses a single landmark, and with the additional information of not seeing any other landmarks it can limit the area of where it believes it could be. The entropy of the distribution is greatly reduced when negative information is incorporated and the rate of convergence towards the estimated position is increased.

Future work will focus on how negative information can be used for other types of landmarks (e.g. field lines) and other sensors. Performance evaluation will be continued in more complex situations and will probe the possibilities of reducing the number of particles necessary for robust Monte Carlo localization.

\section{ACKNOWLEDGMENTS}

Program code used was developed by the GermanTeam, a joint effort of the Humboldt University of Berlin, University of Bremen, University of Dortmund, and the Technical University of Darmstadt. Source code is available for download at http://www.germanteam.org. Freek Stulp pointed out "Silver Blaze" to us.

\section{REFERENCES}

[1] D. Fox, W. Burgard, F. Dellart, and S. Thrun. Monte Carlo Localization: Efficient Position Estimation for Mobile Robots. In Proc. of AAAI, 1999.

[2] D. Fox, W. Burgard, and S. Thrun. Active Markov Localization for Mobile Robots. In Robotics and Autonomous Systems, 1998.

[3] J.-S. Gutmann and D. Fox. An Experimental Comparison of Localization Methods Continued. Proceedings of the 2002 IEEE/RSJ International Conference on Intelligent Robots and Systems (IROS), 2002.

[4] J. Hoffmann, M. Jüngel, and M. Lötzsch. A Vision Based System for Goal-Directed Obstacle Avoidance. In 8th International Workshop on RoboCup 2004 (Robot World Cup Soccer Games and Conferences), Lecture Notes in Artificial Intelligence. Springer, 2005.

[5] W. Koch. On Negative Information in Tracking and Sensor Data Fusion. In Proceedings of the Seventh International Conference on Information Fusion, pages 91-98, 2004.

[6] W. Koch. Utilizing Negative Information to Track Ground Vehicles Through Move-stop-move Cycles. In Proceedings of the SPIE, volume 5429, pages 273-283, 2004.

[7] C. Kwok and D. Fox. Map-based Multiple Model Tracking of a Moving Object. In 8th International Workshop on RoboCup 2004 (Robot World Cup Soccer Games and Conferences), Lecture Notes in Artificial Intelligence. Springer, 2005.

[8] S. Lenser, J. Bruce, and M. Veloso. CMPack: A Complete Software System for Autonomous Legged Soccer Robots. In AGENTS '01: Proceedings of the fifth international conference on Autonomous agents, pages 204-211. ACM Press, 2001.

[9] S. Lenser and M. Veloso. Visual Sonar: Fast Obstacle Avoidance Using Monocular Vision. In Proceedings of IROS'03, 2003.

[10] M. Montemerlo and S. Thrun. Simultaneous Localization and Mapping with Unknown Data Association Using FastSLAM. 2003.

[11] T. Röfer and M. Jüngel. Vision-Based Fast and Reactive Monte-Carlo Localization. In Proceedings of the IEEE International Conference on Robotics and Automation (ICRA-2003), Taipei, Taiwan, pages 856-861, 2003.

[12] S. Särkkä, T. Tamminen, A. Vehtari, and J. Lampinen. Probabilistic Methods in Multiple Target Tracking, Research Report B36. Technical report, Laboratory of Computational Engineering Helsinki University of Technology, 2004.

[13] S. Thrun, W. Burgard, and D. Fox. Probabilistic Robotics, page 231. MIT Press, 2005.

[14] S. Thrun, D. Fox, and W. Burgard. Monte Carlo Localization with Mixture Proposal Distribution. In Proc. of the National Conference on Artificial Intelligence, pages 859-865, 2000. 